\title{
Noninvasive fluorescence monitoring of protoporphyrin IX production and clinical outcomes in actinic keratoses following short-contact application of 5 -aminolevulinate
}

\author{
Christine B. Warren \\ Sara Lohser \\ Lauren C. Wene \\ Cleveland Clinic \\ Department of Dermatology \\ Dermatology and Plastic Surgery Institute \\ 9500 Euclid Avenue \\ Cleveland, Ohio 44195
}

Brian W. Pogue

Dartmouth College

Thayer School of Engineering

8000 Cummings Hall

Hanover, New Hampshire 03755

Philip L. Bailin

Cleveland Clinic

Dermatology and Plastic Surgery Institute

Department of Dermatology

9500 Euclid Avenue

Cleveland, Ohio 44195

\author{
Edward V. Maytin \\ Cleveland Clinic \\ Dermatology and Plastic Surgery Institute \\ and \\ Department of Biomedical Engineering \\ Cleveland, Ohio 44195 \\ and \\ Massachusetts General Hospital \\ Wellman Center for Photomedicine \\ 40 Blossom Street \\ Boston, Massachusetts 02114
}

\begin{abstract}
Topical 5-aminolevulinic acid (ALA) is widely used in photodynamic therapy (PDT) of actinic keratoses (AK), a type of premalignant skin lesion. However, the optimal time between ALA application and exposure to light has not been carefully investigated. Our objective is to study the kinetics of protoporphyrin IX (PpIX) accumulation in AK after short contact ALA and relate this to erythemal responses. Using a noninvasive dosimeter, PpIX fluorescence measurements (5 replicates) were taken at 20-min intervals for $2 \mathrm{~h}$ following ALA application, in 63 AK in 20 patients. Data were analyzed for maximal fluorescent signal obtained, kinetic slope, and changes in erythema. Our results show that PpIX accumulation was linear over time, becoming statistically higher than background in $48 \%$ of all lesions by $20 \mathrm{~min}, 92 \%$ of lesions by $1 \mathrm{~h}$, and $100 \%$ of lesions by $2 \mathrm{~h}$. PplX accumulation was roughly correlated with changes in lesional erythema post-PDT. We conclude that significant amounts of PpIX are produced in all AK lesions by $2 \mathrm{~h}$. The linear kinetics of accumulation suggest that shorter ALA application times may be efficacious in many patients. Noninvasive fluorescence monitoring of PpIX may be useful to delineate areas of high PpIX accumulation within precancerous areas of the skin. (0) 2010 Society of Photo-Optical Instrumentation Engineers. [DOI: 10.1117/1.3484255]
\end{abstract}

Keywords: fiber optic applications; fluorescence; photodetection; photometry; medicine.

Paper 09532SSRR received Dec. 1, 2009; revised manuscript received Apr. 21, 2010; accepted for publication May 19, 2010; published online Sep. 21, 2010.

\section{Introduction}

Photodynamic therapy (PDT) for actinic keratoses (AK), using porphyrin precursors that prime cells to accumulate high levels of intracellular photosensitizer followed by exposure to strong visible light, is now a popular treatment modality worldwide. ${ }^{1}$ In the United States, the combination of 5-aminolevulinate (ALA) and blue light (417 nm) was approved in 1999 for AK, while in Europe, the combination of methyl-aminolevulinate and red light $(\sim 630 \mathrm{~nm})$ is now widely employed for both $\mathrm{AK}$ and nonmelanoma skin cancers. ${ }^{2,3}$ However, the question of how long before irradiation these precursor drugs must be applied in order to obtain a useful level of photosensitizer and a satisfactory response to PDT has not been completely answered. For example, the

Address all correspondence to: Edward Maytin, MD PhD, Desk A61, Dermatology, Cleveland Clinic, 9500 Euclid Avenue, Cleveland, Ohio 44195. Tel: 216445-6676; E-mail: maytine@ccf.org original FDA approval of PDT for the treatment of nonhypertrophic AK of the face and scalp specified a 14- to 18-h drug incubation time after application of 20\% ALA (Levulan Kerastick; DUSA Pharmaceuticals, Inc., Wilmington, Massachusetts), followed by irradiation with $417-\mathrm{nm}$ blue light at $10 \mathrm{~J} / \mathrm{cm}^{2}$ for $1000 \mathrm{~s}$ (Ref. 4). However, because patients must make two office visits (on the first day for drug application, and on the second day for light exposure), this regimen was inconvenient. When Touma et al. ${ }^{5}$ demonstrated in 2004 that shorter ALA incubation times $(1,2$, or $3 \mathrm{~h}$ ) were effective for treatment of $\mathrm{AK}$, clinicians began experimenting informally with reduced contact times in their practices. Many now routinely employ a 2-h drug-light interval, and therapeutic efficacy has been reported with even shorter contact times, for example after 30, 45, or 60 min (Refs. 6-8). However, the biological rationale for PDT using short-contact times remains

$1083-3668 / 2010 / 15(5) / 051607 / 8 / \$ 25.00$ ○ $2010 \mathrm{SPIE}$ 
an open question. Mechanisms by which efficacy under shortcontact conditions may occur have not been established, and the question of how much intracellular PpIX is necessary to achieve therapeutic destruction of preneoplastic cells has not been adequately addressed.

In this report, in situ fluorescence measurements of photosensitizer (PpIX) levels were used to address the hypothesis that changes in PpIX in AK lesions after short-contact ALA application are predictive of cutaneous responses to PDT. Erythema after irradiation was assessed as the primary clinical outcome; the question of whether erythema can be correlated with rates of PpIX accumulation, maximum PpIX attained, or effectiveness of photobleaching was addressed.

\section{Patients/Methods}

\subsection{Study Design}

This small observational study of $63 \mathrm{AK}$ in 20 patients was designed to measure relative changes in PpIX levels in the skin following application of ALA under routine clinical conditions. The study was approved by the Institutional Review Board of the Cleveland Clinic and conducted according to the principles of the Declaration of Helsinki. Interested patients received verbal and written information about the study prior to the first visit; signed informed consent was obtained. The study duration was 4 weeks, including the treatment visit and a 1-month follow-up visit.

\subsection{Patients: Inclusion and Exclusion Criteria}

Males and females with nonhypertrophic AK of the face or scalp were eligible. AK lesions were clinically defined as scaly patches of skin that were distinctly rough to palpation. Patients who were pregnant, nursing, or using any topical treatment for AK were excluded. Immunosuppressed patients on systemic steroids, cyclosporine, or mycophenolate mofetil were allowed in the study, although only one such patient was actually enrolled.

\subsection{Treatment and Measurement Procedures}

On the day of PDT, the patient's face or scalp was lightly cleansed with alcohol, and three AKs in different regions (i.e., left face, forehead, right face) were identified and outlined with a pen. Areas adjacent to each lesion (perilesional; $\sim 0.5$ to $1 \mathrm{~cm}$ away from the AK center), and further away (distant; within $2 \mathrm{~cm}$ of the center) were also selected and photographed. Once the zones were selected, surface PpIX measurements were taken using a hand-held fiber optic-based fluorescence dosimeter (described more fully later). Then, Levulan Kerastick (aminolevulinic acid $\mathrm{HCl}$ for topical solution, 20\%; DUSA Pharmaceuticals, Wilmington, Massachusetts) was applied to the entire face or scalp. Within $5 \mathrm{~min}$, another set of dosimeter measurements was taken (defined as time zero). Measurements were repeated every $20 \mathrm{~min}$, out to $120 \mathrm{~min}$. The skin was then gently wiped with a damp washcloth to remove remaining ALA, the patient was given protective eyewear, positioned in front of the Blu-U light source (DUSA Pharmaceuticals; $\sim 417 \mathrm{~nm}$ ), and $1000 \mathrm{~s}$ of light exposure $\left(10 \mathrm{~J} / \mathrm{cm}^{2}\right)$ was administered. Cold air, cold ultrasound gel, and cool washcloths were used for pain relief as described in Ref. 9. After irradiation, photos were taken of the

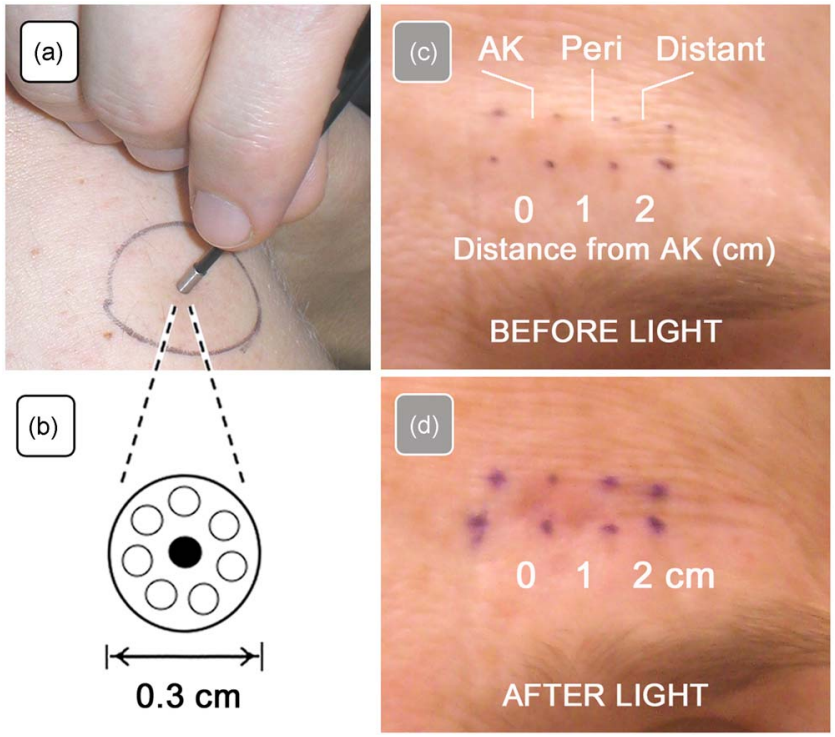

Fig. 1 (a) Fluorescence dosimeter probe applied to patient skin during measurement. (b) Close-up of probe tip, viewed end-on. The central optical fiber carries excitation light $(405 \mathrm{~nm})$ to the skin, and the peripheral optical fibers carry the fluorescent PpIX emissions back to the detector. (c) Close-up of patient's forehead prior to illumination with blue light, to illustrate the relative locations of the clinical lesion $(\mathrm{AK})$, the immediate perilesional zone (Peri), and a distal region (Distant) that were selected for fluorescence measurements. (d) Appearance of same forehead area following $2 \mathrm{~h}$ of topical 5-ALA and a 1000-s exposure to blue light. Note that the clinically selected AK lesion and also the perilesional skin nearby show bright red erythema. (Color online only.)

three previously marked areas. A final set of dosimeter readings was taken within 5 min post-irradiation to assess how much photobleaching of PpIX had occurred. At $48 \mathrm{~h}$ posttreatment, a telephone call to the patient was made to identify any problems or concerns. Patients returned to clinic at one month to assess general clinical response and the need for any additional treatment.

The fluorescence dosimeter used in this study was developed as described in Refs. 10-12, and is manufactured by Aurora Optics, Inc. (Hanover, New Hampshire). A 405-nm-wavelength laser diode sends excitation light down a single 100-micron-diam quartz optical fiber to the skin; fluorescent light is collected and returned to a photomultiplier tube via a ring of seven optical fibers surrounding the excitation fiber [Figs. 1(a) and 1(b)]. Fluorescence is optically filtered to detect only wavelengths above $690 \mathrm{~nm}$, eliminating the excitation light and emission from PpIX photobleaching product fluorescence. For data acquisition, a laptop computer running a customized LabView software program (National Instruments, Inc.) initiates the laser with intensity modulation at $200 \mathrm{~Hz}$, allowing signal detection at this frequency, thereby reducing effects of $1 / f$ noise and $60-\mathrm{Hz}$ optical interference from room lights; the emission signal was sampled at $10-\mathrm{kHz}$ rate for 1-s sampling periods. This was routinely repeated five times to allow for assessment of the stability of the signal. The average power delivered to the tissue is $1 \mathrm{~mW}$, an energy of $1 \mathrm{~mJ}$ per 1-s sample period, which has been found in previous studies to cause little photobleaching of the PpIX. The 
Table 1 Changes in PpIX fluorescence (maximal levels attained, and rates of accumulation) within AK lesions from twenty ${ }^{\mathrm{a}}$ patients during the first $2 \mathrm{~h}$ after topical 5-ALA.

\begin{tabular}{|c|c|c|c|c|c|c|c|}
\hline \multirow{2}{*}{$\begin{array}{l}\text { Patient } \\
\text { identifier }\end{array}$} & \multirow[b]{2}{*}{ Sex } & \multirow[b]{2}{*}{ Age (yr) } & \multirow{2}{*}{$\begin{array}{l}\text { Lesion } \\
\text { location }\end{array}$} & \multicolumn{2}{|c|}{ Change in signal $\left(\Delta F_{\max }\right)$} & \multicolumn{2}{|c|}{ Kinetic slope $(\times 1000)$} \\
\hline & & & & Mean & S.D. & Mean & S.D. \\
\hline A & $\mathrm{m}$ & 74 & Scalp & 1.82 & 1.1 & 16.67 & 13.2 \\
\hline B & f & 60 & Face & 1.95 & 0.1 & 29.67 & 22.0 \\
\hline C & $f$ & 65 & Face & 2.32 & 0.8 & 19.00 & 7.9 \\
\hline D & $\mathrm{m}$ & 74 & Face & 2.44 & 2.3 & 20.00 & 16.5 \\
\hline E & $\mathrm{m}$ & 61 & Face & 2.49 & 3.5 & 16.33 & 33.1 \\
\hline $\mathrm{F}$ & f & 75 & Face & 2.51 & 0.9 & 21.00 & 9.0 \\
\hline G & $f$ & 72 & Face & 2.96 & 2.3 & 24.33 & 16.6 \\
\hline $\mathrm{H}$ & f & 46 & Face & 4.04 & 3.4 & 40.67 & 35.4 \\
\hline I & $\mathrm{m}$ & 71 & Face & 4.07 & 5.0 & 29.00 & 35.7 \\
\hline J & $f$ & 79 & Face & 4.43 & 1.0 & 41.67 & 10.0 \\
\hline K & $\mathrm{m}$ & 55 & Scalp & 4.77 & 1.7 & 33.33 & 12.7 \\
\hline L & $\mathrm{m}$ & 52 & Scalp & 5.17 & 2.2 & 39.67 & 11.6 \\
\hline M & $\mathrm{m}$ & 78 & Scalp & 5.31 & 3.7 & 40.67 & 31.8 \\
\hline $\mathrm{N}$ & $f$ & 55 & Face & 5.64 & 2.2 & 49.67 & 16.2 \\
\hline O & $f$ & 60 & Face & 5.86 & 4.1 & 50.67 & 37.7 \\
\hline P & $\mathrm{m}$ & 71 & Scalp & 6.24 & 4.9 & 59.00 & 38.7 \\
\hline Q & $\mathrm{m}$ & 62 & Scalp & 6.41 & 8.1 & 54.00 & 89.4 \\
\hline $\mathrm{R}$ & $f$ & 71 & Face & 6.73 & 1.9 & 55.00 & 28.8 \\
\hline S & $\mathrm{m}$ & 76 & Face & 6.82 & 1.9 & 65.67 & 12.9 \\
\hline $\mathrm{T}$ & $\mathrm{m}$ & 55 & Face & 7.43 & 1.7 & 55.67 & 8.6 \\
\hline U & $\mathrm{m}$ & 65 & Face & 10.93 & 4.4 & 88.00 & 22.9 \\
\hline
\end{tabular}

Patients are arranged in order of ascending value of their mean $\Delta F_{\text {max }}$.

Each value is an average of three AK lesions per patient.

apatients $K$ (scalp) and $T$ (face) are the same individual.

five repeated measurements show significantly less than $10 \%$ photobleaching during a single acquisition.

The system was calibrated for intensity before each use; semiautomated measurements are taken on a black negative fluorescence standard, followed by a blue positive fluorescence standard made from rubberized foam. With these data, daily variations in response are automatically normalized, allowing quantitative comparison of measurements between acquisition sessions. Data, in standard fluorescence units, are stable because of the calibration process but do not have specific units associated with them.

\subsection{Analysis of PpIX Fluorescence}

Fluorescence dosimeter readings were in arbitrary units (AU). Maximal increases in PpIX fluorescence $\left(\Delta F_{\max }\right)$, reported in
Tables 1 and 2, were calculated by subtracting time-zero readings from the 2 - $\mathrm{h}$ readings. Rates of PpIX production were determined from the fluorescence versus time curves (AU/ $\min$ ) for each lesion.

\subsection{Analysis of Photobleaching}

Photobleaching (PB) from the light treatment, which reflects the relative loss of fluorescent signal due to destruction of PpIX molecules during exposure to light, was calculated by taking a last fluorescence measurement immediately postirradiation, correcting it by subtracting out the time-zero background, dividing this difference by $\Delta F_{\text {max }}$, and subtracting that value from 1. PB is quantified as a percentage and is reported in Table 2. 
Warren et al.: Noninvasive fluorescence monitoring of protoporphyrin IX production...

Table 2 Clinical erythema responses in AK lesions, in relation to maximal change in fluorescence $\left(\Delta F_{\text {max }}\right)$ and extent of photobleaching (PB).

\begin{tabular}{|c|c|c|c|c|c|}
\hline \multirow[b]{2}{*}{ Lesion* } & \multirow[b]{2}{*}{$\Delta F_{\max }$} & \multirow[b]{2}{*}{$\begin{array}{l}\mathrm{PB} \\
(\%)\end{array}$} & \multicolumn{3}{|c|}{ Erythema assessment } \\
\hline & & & Change & 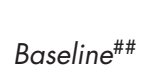 & Combo \\
\hline El & 0.3 & 100 & 0 & 0 & 0 \\
\hline D1 & 0.4 & $3.7^{* *}$ & 0 & 1 & 1 \\
\hline G1 & 0.5 & $50 * *$ & 0 & 1 & 1 \\
\hline E2 & 0.7 & $39 * *$ & 1 & 1 & 2 \\
\hline 11 & 0.9 & $49 * *$ & 0 & 0 & 0 \\
\hline $\mathrm{Pl}$ & 1.1 & 83 & 0 & 1 & 1 \\
\hline Q1 & 1.2 & $38^{* *}$ & 1 & 1 & 2 \\
\hline $\mathrm{Fl}$ & 1.4 & 96 & 0 & 1 & 1 \\
\hline 12 & 1.5 & 98 & 1 & 0 & 0 \\
\hline $\mathrm{Hl}$ & 1.5 & $52^{* *}$ & 0 & 0 & 0 \\
\hline $\mathrm{Cl}$ & 1.7 & $50 * *$ & 1 & 1 & 2 \\
\hline B1 & 1.9 & $53^{* *}$ & 1 & 1 & 2 \\
\hline B2 & 1.9 & 63 & 0 & 0 & 0 \\
\hline D2 & 1.9 & 100 & 0 & 1 & 1 \\
\hline $\mathrm{C} 2$ & 2.1 & $38^{* *}$ & 1 & 1 & 2 \\
\hline B3 & 2.1 & 64 & 1 & 0 & 1 \\
\hline Q2 & 2.3 & 98 & 1 & 1 & 2 \\
\hline 01 & 2.6 & $58^{* *}$ & 1 & 1 & 2 \\
\hline $\mathrm{H} 2$ & 2.7 & 80 & 0 & 0 & 0 \\
\hline $\mathrm{LI}$ & 3.0 & $53^{* *}$ & 0 & 1 & 1 \\
\hline F2 & 3.0 & 83 & 0 & 1 & 1 \\
\hline F3 & 3.1 & 83 & 0 & 1 & 1 \\
\hline $\mathrm{C} 3$ & 3.2 & 67 & 0 & 1 & 1 \\
\hline G2 & 3.3 & 100 & 0 & 1 & 1 \\
\hline $\mathrm{Kl}$ & 3.6 & 84 & 0 & 1 & 1 \\
\hline $\mathrm{J}$ & 3.6 & $61^{* *}$ & 1 & 1 & 2 \\
\hline$M$ & 3.9 & 75 & 1 & 1 & 2 \\
\hline $\mathrm{K} 2$ & 3.9 & $47^{* *}$ & 1 & 1 & 2 \\
\hline $\mathrm{J} 2$ & 4.2 & $62^{* *}$ & 1 & 1 & 2 \\
\hline O2 & 4.5 & 76 & 1 & 0 & 1 \\
\hline $\mathrm{R} 1$ & 4.6 & 97 & 0 & 1 & 1 \\
\hline D3 & 5.0 & 68 & 0 & 1 & 1 \\
\hline $\mathrm{S} 1$ & 5.0 & $55^{* *}$ & 1 & 1 & 2 \\
\hline G3 & 5.1 & 99 & 0 & 1 & 1 \\
\hline L2 & 5.3 & 91 & 1 & 1 & 2 \\
\hline J3 & 5.5 & 80 & 1 & 1 & 2 \\
\hline $\mathrm{Tl}$ & 6.0 & 100 & 1 & 1 & 2 \\
\hline E3 & 6.6 & 94 & 1 & 1 & 2 \\
\hline S2 & 6.8 & 84 & 1 & 1 & 2 \\
\hline K3 & 6.8 & 65 & 1 & 1 & 2 \\
\hline $\mathrm{T} 2$ & 6.9 & 86 & 1 & 0 & 1 \\
\hline P2 & 7.0 & 91 & 0 & 1 & 1 \\
\hline L3 & 7.3 & 66 & 1 & 1 & 2 \\
\hline R2 & 7.3 & 84 & 0 & 1 & 1 \\
\hline $\mathrm{H} 3$ & 7.9 & 90 & 1 & 0 & 1 \\
\hline U1 & 8.1 & 79 & 1 & 1 & 2 \\
\hline R3 & 8.3 & 97 & 1 & 1 & 2 \\
\hline U2 & 8.7 & 88 & 1 & 1 & 2 \\
\hline S3 & 8.7 & 74 & 1 & 1 & 2 \\
\hline T3 & 9.4 & 91 & 1 & 1 & 2 \\
\hline
\end{tabular}


Table 2 (Continued.)

\begin{tabular}{cccccc}
\hline & & \multicolumn{3}{c}{ Erythema assessment } \\
\cline { 4 - 6 } Lesion $^{*}$ & $\Delta F_{\text {max }}$ & $(\%)$ & Change $^{\#}$ & Baseline $^{\# \#}$ & Combo $^{a}$ \\
\hline M & 9.6 & 82 & 1 & 1 & $\mathbf{2}$ \\
I3 & 9.8 & 99 & 1 & 1 & $\mathbf{2}$ \\
O3 & 10.5 & 82 & 1 & 1 & $\mathbf{2}$ \\
P3 & 10.7 & 98 & 0 & 1 & $\mathbf{2}$ \\
Q3 & 15.8 & 89 & 1 & 1 & $\mathbf{2}$ \\
U3 & 16.0 & 99 & 1 & 1 & \\
\hline
\end{tabular}

Values are for individual lesions, arranged in ascending order of $\Delta F_{\max }$.

* Individual AK, identified by patient (A to $U$ ) and lesion $(1,2$, or 3 ).

** Photobleaching value less than $63 \%$ (see text).

\# Change in erythema: 1 if erythema was increased after PDT, 0 if unchanged after PDT.

\#\# Baseline erythema: 1 if erythema was present before PDT, 0 if absent before PDT.

a Erythema combined score, sum of the two preceding columns.

\subsection{Analysis of Erythema}

Erythema, defined as the presence of a red color due to blood vessel dilation in the skin, was scored from clinical photographs for each AK lesion by two independent observers who were blinded as to when the photographs had been taken. Two parameters were evaluated, each in a binary fashion $(0=$ no, $1=$ yes), by using the photographs to answer the following questions: (1) Did the lesion show erythema at baseline? (2) Was there a visible increase in erythema following PDT? Also, to improve the discriminatory power of the data, the numerical answers from questions 1 and 2 were added together to create an erythema combined score (ECS), compiled in Table 2 and Fig. 2; see Sec. 3 for further details.

\section{Results and Discussion}

Twenty patients, already scheduled to undergo routine ALAPDT for actinic keratoses treatment were serially enrolled in this pilot study to monitor rates of PpIX accumulation in AK

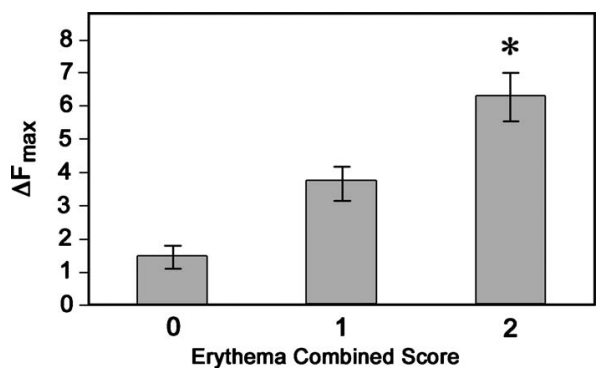

Fig. 2 Correlation between erythema and the maximal increase in PplX fluorescence $\left(\Delta F_{\text {max }}\right)$ at $2 \mathrm{~h}$ after ALA application. An erythema combined score (ECS) was determined from 56 lesions available for analysis and plotted versus $\Delta F_{\max }$ from the data in Table 2. An ANOVA (one-way between subjects) was performed to compare ECS and $\Delta F_{\max }$ using the Analyse-it v. 2.20 add-in software for Microsoft Excel. Here, a significant effect of erythema on $\Delta F_{\max }$ at the $p<0.05$ level was shown $(*)$ for the three groups $[F(2,58)=8.00, p=0.0009]$. Post hoc comparisons using the Tukey range test indicated that $\Delta F_{\max }$ values for an ECS of $2(M=6.27, \mathrm{SD}=3.91)$ versus $\mathrm{ECS}$ of 0 $(M=1.42, \mathrm{SD}=0.83)$ were significantly different, as were $\Delta F_{\max }$ values for ECS of 2 versus ECS of $1(M=3.65, S D=2.57)$. lesions on the face or scalp (Table 1). Ages of the patients ranged from 52 to 79 (mean 65.6 years), and nine patients $(45 \%)$ were women. For the 21 ALA-PDT study sessions, a total of 63 lesions and 126 adjacent sites were measured. An example of how noninvasive measurements were done is illustrated in Fig. 1. Although data were collected from both within and around each AK lesion, our analysis for this report will deal primarily with the PpIX produced within lesions. A preliminary analysis of perilesional effects is discussed at the end of this section.

\subsection{Noninvasive Monitoring Shows Linear Rates of Photosensitizer Accumulation in AK Lesions}

Figure 3 illustrates the development of PpIX signals in AK lesions as a function of time after ALA application, displayed either as absolute fluorescence readings in a single lesion [Fig. $3(\mathrm{a})$ ] or as normalized readings from multiple patients [Fig. 3(b)]. In the latter example [Fig. 3(b)], readings from all lesions on the face (45 AK, from 15 patients) were combined after normalization to the 2-h readings (set at $100 \%$ ), following background subtraction. With either type of plot, the linearity of PpIX accumulation of PpIX over the $2 \mathrm{~h}$ of contact with ALA was evident, and linearity was observed for all but a few of the 63 lesions studied. No significant differences in lesional PpIX accumulation were observed for face versus scalp.

PpIX accumulation between different lesions varied widely. When expressed as a difference of PpIX readings taken pre- and post-ALA application, the change in fluorescence among different lesions varied from no change to an increase of greater than $10 \mathrm{~A} . \mathrm{U}$. at $2 \mathrm{~h}$ relative to baseline [Fig. 3(c)]. High variability was observed even after subtraction of the background (non-PpIX-related signal at zero time), as can be seen from the large standard deviations in $\Delta F_{\text {max }}$ in Table 1 (column 6). While this large variability probably reflects several factors, including the biological heterogeneity among lesions, it is interesting that the data do tend to cluster in characteristic patterns in some patients. For example, from Table 1, the mean PpIX signal attained in the three AK lesions on the skin of patient $U\left(\Delta F_{\max } 10.93 \pm 4.4\right)$ were all significantly higher than the three lesions on patient $\mathrm{D}$ 


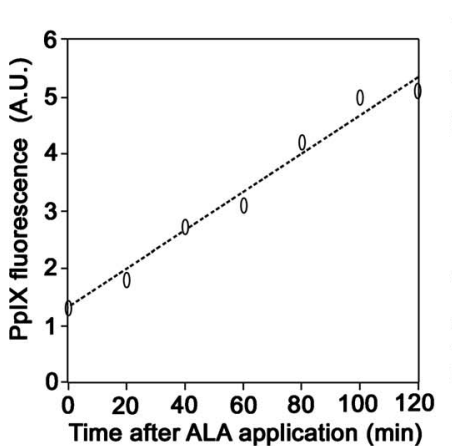

(a)

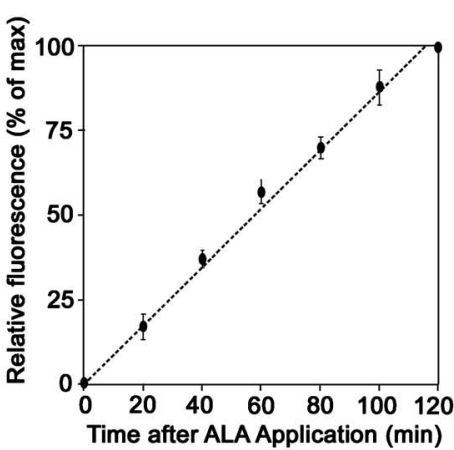

(b)

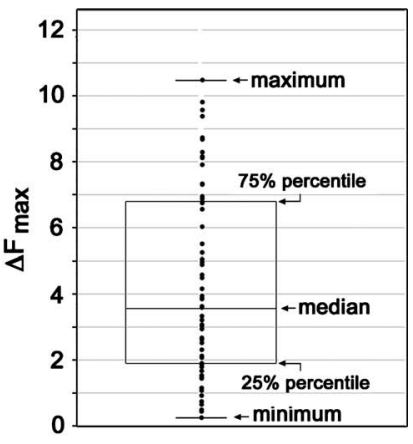

(c)

Fig. 3 (a) Example of raw data for an AK lesion on the left cheek of Patient J. Fluorescent PpIX signal as a function of time after ALA application, without background subtraction. Each point is the mean of five readings \pm SD; readings are expressed in arbitrary units (A.U.). (b) Summary of readings taken from AK lesions of the face (15 patients, 45 lesions). Data are normalized to the maximal fluorescence reading in each patient, after background subtraction ( $n=45$; mean \pm SEM). (c) Box-and-whisker plot to illustrate variability in maximal PpIX accumulation in 60 AK on the face at $2 \mathrm{~h}$ after ALA application. PpIX signals on the $y$ axis represent the change in fluorescence between $2 \mathrm{~h}$ and time zero (i.e., 2 - $\mathrm{h}$ fluorescence readings with background subtraction). Circles represent individual lesions.

$\left(\Delta F_{\max } 2.44 \pm 2.3\right)$, suggesting perhaps a fundamental difference in physiology between the two individuals (among other explanations). The kinetics of PpIX accumulation within AK lesions followed a similar rank-order as the $\Delta F_{\max }$ values and showed similar variability (Table 1 , column 7).

An important question, in terms of predicting therapeutic selectivity, is whether PpIX accumulates more in preneoplastic skin (AK lesions) than in normal skin. In normal facial skin of five subjects (from an area under the chin), the mean $\Delta F_{\text {max }}$ measured at $2 \mathrm{~h}$ after ALA treatment was $1.80 \pm 0.80$. Patient $\mathrm{A}$ in Table 1 had nearly the same mean $\Delta F_{\max }$ value (1.82), and patients B to I, while displaying mean $\Delta F_{\text {max }}$ values higher than normal skin, were statistically indistinguishable from the normal skin readings. This suggests that the use of aggregated values of $\Delta F_{\max }$ to predict lesion-selective behavior may not be very useful. However, an examination of PpIX fluorescence in individual lesions is more helpful (Table $2)$. The AK lesions in Table 2 have been arranged in ascending order of $\Delta F_{\max }$ values. Knowing that $\Delta F_{\max }$ in normal skin is $1.80 \pm 0.80$, one can see from Table 2 that the lowest one-third of $\Delta F_{\max }$ values (column 2) fall below or within this range. If one uses $\Delta F_{\max }$ of normal skin, plus two standard deviations, as a cut-off to define a "significant increase" in PpIX levels, then AK lesions with an $\Delta F_{\text {max }}$ of 3.4 or higher could be considered significantly elevated.

\subsection{Photobleaching in AK Lesions}

In Table 2, it can be noted that the extent of photobleaching (PB) seems to correlate with $\Delta F_{\max }$. To formally determine whether photobleaching is significantly less efficient for lesions with a low PpIX signal, a formal statistical analysis confirmed that for AK lesions with $\Delta F_{\max }<2.0$, the average photobleaching $(\mathrm{PB}=63 \%)$ is significantly less than for lesions with $\Delta F_{\max }$ greater than $2.0(\mathrm{~PB}=78 \%$; student's $t$-test, two-tailed, assuming equal variance, $P$ value of 0.008$)$. PB values less than $63 \%$ are indicated with two asterisks in Table 2. Why this should be the case remains unknown, but one possible explanation could be that lesions with low $\Delta F_{\max }$ and
PB values are less optically transparent than other lesions, due for example to the presence of a thick hyperkeratotic scale overlying the lesion.

\subsection{Erythema Correlated with the Amount of PpIX Synthesized}

Because erythema can be regarded as a surrogate biological readout of photodynamic effects in the skin, the relationship between PpIX levels, photobleaching, and erythema within each individual AK lesion was examined (Table 2). As described in Sec. 2, the degree of redness (erythema) was evaluated from clinical photographs and rated for increases in erythema induced by PDT (Table 2, column 4). A trend between $\Delta F_{\text {max }}$ and increased erythema was noted, but this was not statistically significant. Also evaluated was whether AK showed erythema at baseline, to test the hypothesis that more severely dysplastic and inflamed AK lesions might be more responsive to PDT (Table 2, column 5). Again, a weak trend between $\Delta F_{\max }$ and erythema was noted. Interestingly, by adding the two erythema parameters together to create a combined score (Table 2, column 6), a clear-cut relationship between PpIX levels and erythema emerged, with the erythema combined score significantly correlated with $\Delta F_{\max }$ (Fig. 2).

Regarding therapeutic responses, this study was not designed to determine long-term clinical outcomes. At the 1-month follow-up visit, 7 of 20 patients had one or more detectable AK. Whether these lesions represented treatment failures or new AKs could not be determined because the study protocol was not designed to specifically follow the three lesions measured initially in each patient.

\subsection{Majority of AKs Demonstrate Significant PpIX Accumulation within 1 Hour}

Despite the possibility of a few nonresponsive lesions, the vast majority of the $63 \mathrm{AKs}$ resolved at 1 month post-PDT, regardless of their $\Delta F_{\max }$ status. This illustrates the following conundrum for this type of clinical study: one simply does not know how much PpIX is sufficient to trigger lesion resolution since the biology of the PDT response (immune responses and 
Table 3 Duration of 5-ALA exposure required for PpIX accumulation in AK lesions. Proportion of lesions that reached the fluorescence threshold at the times indicated.

\begin{tabular}{|c|c|c|c|c|c|c|c|}
\hline & \multicolumn{6}{|c|}{ Time (min) for fluorescence to reach threshold } & \multirow[b]{3}{*}{ Total: } \\
\hline & 20 & 40 & 60 & $80-99$ & $100-119$ & $>120$ & \\
\hline Threshold: & & & & & & & \\
\hline Baseline + 1 S.D. & $47.6 \%$ & $28.5 \%$ & $15.9 \%$ & $6.4 \%$ & $1.6 \%$ & $0.0 \%$ & $100 \%$ \\
\hline Baseline+3 S.D. & $3.2 \%$ & $25.4 \%$ & $17.5 \%$ & $17.5 \%$ & $12.6 \%$ & $23.8 \%$ & $100 \%$ \\
\hline
\end{tabular}

cell death pathways) remains incompletely understood. Therefore, the data were evaluated from a reverse perspective. Assuming that detectable increases in PpIX $\left(\Delta F_{\max }\right)$, no matter how small, were sufficient to generate a clinically significant PDT effect (because essentially all lesions in the study resolved after PDT), the ability of noninvasive monitoring to first detect a change in fluorescence within the AK lesions was evaluated. As an objective threshold to define "time to first increase," the variability (S.D.) of the five repeated measurements taken from the lesion at any given time point was employed. (Photobleaching during repeated measurements was found to be insignificant.) A threshold was set, and the proportion of AK lesions that successfully crossed that threshold was evaluated using a student's $t$-test to compare measurements at the test time versus zero time $(p<0.05$ significance) for each time point (Table 3). If the threshold was defined as one standard deviation above the time-zero baseline, then within $20 \mathrm{~min}$ after topical ALA application, half of all lesions $(48 \%)$ showed a statistically significant increase in PpIX fluorescence. Essentially all $(98.4 \%)$ did so by $2 \mathrm{~h}$. Using a more stringent threshold, 3 S.D. above baseline, half of all lesions $(46.1 \%)$ reached the threshold value within the first hour (Table 2, row 2).

\subsection{Noninvasive Fluorescence Monitoring to Detect Field Cancerization Effects}

While only the intralesional PpIX data have been rigorously analyzed, measurements from perilesional and distant sites [as in Fig. 1(c)] were collected. A preliminary description of the typical patterns observed is given in Fig. 4. As a function of the distance from the center of each AK (up to $2 \mathrm{~cm}$ away), PpIX measurements yielded three overall patterns (Fig. 4). Half $(49 \%)$ of the 63 zones around the $63 \mathrm{AK}$ in the study showed highest readings within the lesion and lower readings at the periphery (Fig. 4, pattern a). Another $28 \%$ of regions displayed an extended pattern (Fig. 4, pattern b) in which perilesional readings were very similar to the intralesional reading. Pattern b might be explained by the "field cancerization," a clinically well-accepted phenomenon in which preneoplastic changes within the skin extend well beyond the clinically detectable lesion. ${ }^{13}$ Last, $23 \%$ of regions had lower readings within the lesion than in the surrounding skin (Fig. 4, pattern c), although these readings were still higher than readings from normal skin or photobleached lesions (Fig. 4, zone shown in gray). Pattern c might be explained by attenuation of the fluorescence signal due to enhanced scattering (for example, from scale on the AK lesion's surface).

\section{Conclusions}

PDT is now a popular modality for treatment of AK, and physicians have tried to enhance convenience for the patient by shortening the duration of ALA application from the originally recommended $14-18 \mathrm{~h}$ to $2 \mathrm{~h}$ or less, but without any scientific justification. To address this lack of evidence, a detailed kinetic study of PpIX accumulation in AK lesions and adjacent skin during the first $2 \mathrm{~h}$ following topical application of ALA was performed, using noninvasive fluorescence moni-

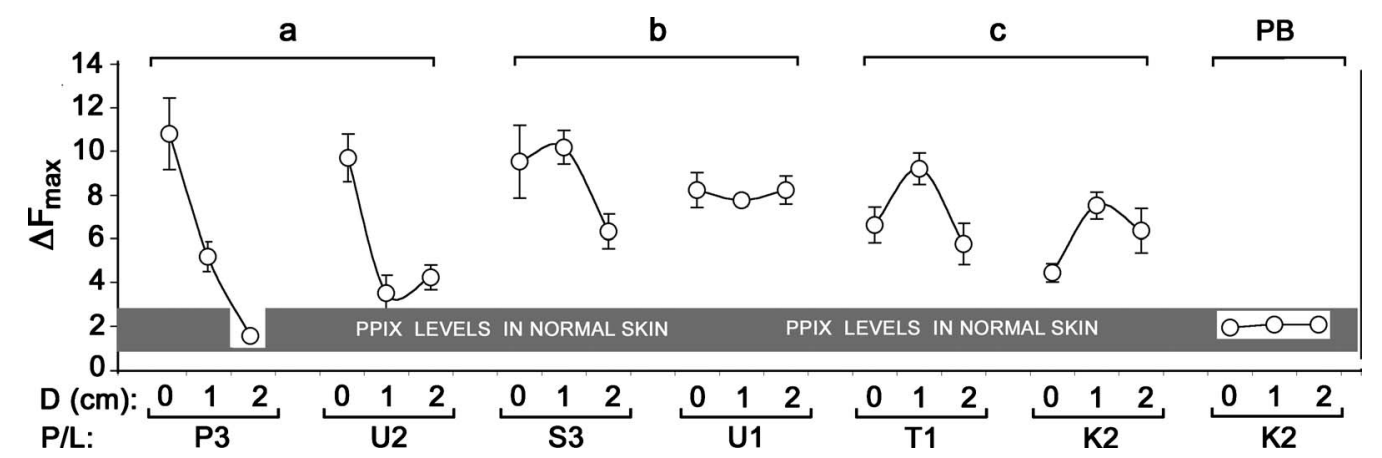

Fig. 4 Regional patterns of PpIX-specific fluorescence (see text for further explanation). $\Delta F_{\text {max }}$ fluorescence at $2 \mathrm{~h}$ after ALA application, measured at location specified by D. D-distance from the center of the lesion $(0,1$, or $2 \mathrm{~cm})$. P/L-identifier for patient/lesion from Table 2 . Gray area-range of $\Delta F_{\max }$ in normal skin. $\mathrm{PB}$ - photobleached. 
toring to measure PpIX in patients undergoing PDT under routine clinical conditions. From the data, the following conclusions can be drawn:

1. Within the 2-h period examined, PpIX accumulated in a linear fashion. The PpIX signal reached a threshold of one standard deviation (S.D.) above baseline in half of all lesions within the first 20 min of ALA contact time, and in all lesions by $2 \mathrm{~h}$.

2. With a more stringent threshold criterion, $46 \%$ of lesions reached 3 S.D. above baseline by $1 \mathrm{~h}$, and $75 \%$ did so by $2 \mathrm{~h}$. Essentially all of the AK lesions showed clinical resolution after this PDT regimen, so the PpIX levels measured in the lesions were biologically significant, by definition.

3. As a more proximal means to measure the biological effects of PDT, the amount of erythema in AK lesions immediately post-PDT was found to correlate with PpIX accumulation, similar to previous observations of a positive correlation between PpIX levels and postPDT erythema in normal skin. ${ }^{14,15}$

4. Elevated PpIX levels were demonstrated in areas of photodamaged skin that appeared otherwise normal (the field cancerization effect). While fluorescence imaging of PpIX has been proposed as a method to define the borders of skin tumors prior to surgical removal, ${ }^{16-19}$ our findings suggest an additional potential use. Because the conversion of ALA to PpIX reflects changes in metabolic activity within neoplastic cells, ${ }^{13,20}$ PpIX fluorescence imaging should be further explored as a technique to identify epidermal regions that harbor precancerous changes that might otherwise go undetected.

\section{Acknowledgments}

We thank Jim Sherer, P.A., and Fran Moore, P.A., for allowing us to coordinate research visits with their PDT clinic patient schedules. Grant support comes from the National Institutes of Health/National Cancer Institute Grant No. P01 CA084203 (T. Hasan, E. Maytin, B. Pogue). The authors state no conflicts of interest to disclose.

\section{References}

1. M. A. MacCormack, "Photodynamic therapy," Adv. Dermatol. 22, 219-258 (2006).

2. L. R. Braathen, R. M. Szeimies, N. Basset-Seguin, R. Bissonnette, P. Foley, D. Pariser, R. Roelandts, A. M. Wennberg, and C. A. Morton, "Guidelines on the use of photodynamic therapy for nonmelanoma skin cancer: an international consensus. International Society for Photodynamic Therapy in Dermatology, 2005," J. Am. Acad. Dermatol. 56(1), 125-143 (2007).

3. J. E. Blume and A. R. Oseroff, "Aminolevulinic acid photodynamic therapy for skin cancers," Dermatol. Clin. 25(1), 5-14 (2007).

4. DUSA Pharmaceuticals, "Levulan Kerastick topical solution 20\%," package insert 〈http://www.dusapharma.com〉 (accessed June 7, 2009).
5. D. Touma, M. Yaar, S. Whitehead, N. Konnikov, and B. A. Gilchrest, "A trial of short incubation, broad-area photodynamic therapy for facial actinic keratoses and diffuse photodamage," Arch. Dermatol. 140(1), 33-40 (2004).

6. M. H. Gold, "Photodynamic therapy in dermatology: the next five years," Dermatol. Clin. 25(1), 119-120 (2007).

7. A. Itkin and B. A. Gilchrest, "Delta-aminolevulinic acid and blue light photodynamic therapy for treatment of multiple basal cell carcinomas in two patients with nevoid basal cell carcinoma syndrome," Dermatol. Surg. 30(7), 1054-1061 (2004).

8. K. P. Redbord and C. W. Hanke, "Topical photodynamic therapy for dermatologic disorders: results and complications," J. Drugs Dermatol. 6(12), 1197-1202 (2007).

9. C. B. Warren, L. J. Karai, A. Vidimos, and E. V. Maytin, "Pain associated with aminolevulinic acid-photodynamic therapy of skin disease," J. Am. Acad. Dermatol. 61(6), 1033-1043 (2009).

10. B. W. Pogue and G. Burke, "Fiber-optic bundle design for quantitative fluorescence measurement from tissue," Appl. Opt. 37(31), 7429-7436 (1998)

11. X. Zhou, B. W. Pogue, B. Chen, E. Demidenko, R. Joshi, J. Hoopes, and T. Hasan, "Pretreatment photosensitizer dosimetry reduces variation in tumor response," Int. J. Radiat. Oncol., Biol., Phys. 64(4), 1211-1220 (2006).

12. C. Sheng, P. J. Hoopes, T. Hasan, and B. W. Pogue, "Photobleachingbased dosimetry predicts deposited dose in ALA-PpIX PDT of rodent esophagus," Photochem. Photobiol. 83(3), 738-748 (2007).

13. S. Anand, G. Honari, T. Hasan, P. Elson, and E. V. Maytin, "Lowdose methotrexate enhances aminolevulinate-based photodynamic therapy in skin carcinoma cells in vitro and in vivo," Clin. Cancer Res. 15(10), 3333-3343 (2009).

14. S. Gerscher, J. P. Connelly, G. M. Beijersbergen Van Henegouwen, A. J. MacRobert, P. Watt, and L. E. Rhodes, "A quantitative assessment of protoporphyrin IX metabolism and phototoxicity in human skin following dose-controlled delivery of the prodrugs 5-aminolaevulinic acid and 5-aminolaevulinic acid-n-pentylester," $\mathrm{Br}$. J. Dermatol. 144(5), 983-990 (2001).

15. S. Gerscher, J. P. Connelly, J. Griffiths, S. B. Brown, A. J. MacRobert, G. Wong, and L. E. Rhodes, "Comparison of the pharmacokinetics and phototoxicity of protoporphyrin IX metabolized from 5-aminolevulinic acid and two derivatives in human skin in vivo," Photochem. Photobiol. 72(4), 569-574 (2000)

16. S. Andersson-Engels, G. Canti, R. Cubeddu, C. Eker, C. af Klinteberg, A. Pifferi, K. Svanberg, S. Svanberg, P. Taroni, G. Valentini, and I. Wang, "Preliminary evaluation of two fluorescence imaging methods for the detection and the delineation of basal cell carcinomas of the skin," Lasers Surg. Med. 26(1), 76-82 (2000).

17. R. Na, I. M. Stender, and H. C. Wulf, "Can autofluorescence demarcate basal cell carcinoma from normal skin? A comparison with protoporphyrin IX fluorescence," Acta Derm Venereol 81(4), 246-249 (2001).

18. B. Stenquist, M. B. Ericson, C. Strandeberg, L. Molne, A. Rosen, O. Larko, and A. M. Wennberg, "Bispectral fluorescence imaging of aggressive basal cell carcinoma combined with histopathological mapping: a preliminary study indicating a possible adjunct to Mohs micrographic surgery," Br. J. Dermatol. 154(2), 305-309 (2006)

19. R. Alkalay, J. Alcalay, A. Maly, A. Ingber, C. Fritsch, T. Ruzicka, and C. D. Enk, "Fluorescence imaging for the demarcation of basal cell carcinoma tumor borders," J. Drugs Dermatol. 7(11), 1033-1037 (2008).

20. E. V. Maytin, S. Anand, C. Baran, G. Honari, S. Lohser, A. Kyei, P. Bailin, and B. W. Pogue, "Enhancement and optimization of PpIXbased photodynamic therapy of skin cancer: translational studies from bench to clinic," in Mechanisms and Techniques in Photodynamic Therapy XVIII, D. Kessel, Ed., Proc. SPIE 7164, 71640K (2009). 